\title{
Intervención formativa y seguimientos observacionales con directivos de un hospital en México
}

\section{Formative intervention and observational follow-ups with Directors of a Mexican hospital}

\author{
Brito Rivera, Hugo Armando ${ }^{1}$
}

\begin{abstract}
Resumen:
En el presente artículo se analiza la técnica shadowing como parte de un Laboratorio de Cambio con directivos del Cuerpo de Gobierno de un hospital público. Se realizó un seguimiento observacional tipo shadowing a tres participantes (subdirector del hospital, jefa de epidemiología y subdirectora administrativa) para identificar y exponer habilidades blandas y zonas de desarrollo próximo. Los datos obtenidos, de tipo cualitativo, fueron analizados desde una estrategia multifocal, descriptiva e interpretativa consistente en la codificación y selección de extractos de la transcripción de notas de campo, videograbaciones y documentos de restitución. Se presentan dos macro categorías vinculadas; hallazgos de la observación (habilidades y zonas de desarrollo) y evaluación de la técnica por los participantes (aspectos metodológicos y utilidad). Los resultados indican prácticas discursivas transicionales y significados intermedios inherentes al uso de los seguimientos como práctica de innovación metodológica y actividad formativa no dominante. Se concluye que el shadowing confirmó su pertinencia para identificar y apoyar habilidades profesionales desde la intervención, estimulando agencia, apertura del horizonte de observación, construcción de significados y nuevas prácticas formativas.
\end{abstract}

Palabras Clave: Aprendizaje organizacional, competencias profesionales, shadowing, liderazgo, Laboratorio de Cambio.

${ }^{1}$ Università di Roma La Sapienza, Italia. ORCID: 0000-0003-1363-5980 .

*Correspondencia: hugoarmando.britorivera@gmail.com

$$
\text { . }
$$

This article analyses the shadowing technique as part of a Change Laboratory carried out with the Board of Directors in a public hospital. Three participants (deputy director of the hospital, head of epidemiology and administration deputy head) were followed up with shadowing technique to identify and expose soft skills and zones of proximal development. The qualitative data obtained were analysed using a multifocal, descriptive and interpretative strategy consisting of the coding and selection of extracts from the transcription of field notes, video recordings and feedback documents. Two linked macro categories are presented; observation findings (skills and development zones) and evaluation of the technique by the participants (methodological aspects and usefulness). The outcomes indicate transitional discursive practices and intermediate meanings inherent to the use of follow -ups as a methodological innovation practice and non-dominant training activity. It is concluded that shadowing confirmed its relevance to identify and support professional skills from the intervention, stimulating agency, opening of the observation horizon, construction of meanings and new formative practices.

Keywords: Organizational learning, professional competencies, shadowing, leadership, Change Laboratory. 
Los modelos tradicionales de intervención orientada a la formación continua de directivos en hospitales y otras organizaciones (como es el caso de directivos en empresas) se basan en metodologías de "arriba hacia abajo" que conllevan formas de participación vertical. En contraposición, las metodologías de intervención situada de reciente generación consideran procedimientos de "abajo hacia arriba" en los que la participación se distribuye horizontalmente. De acuerdo con Engeström (2011) las primeras representan metodologías de intervención lineal, mientras que las segundas se denominan en términos de metodologías de intervención formativa.

Las metodologías lineales se caracterizan generalmente por formas de comunicación en las que un experto explica cómo debe realizarse la actividad laboral, abordando problemáticas y proporcionando modelos prescriptivos para la resolución de las mismas. Tales metodologías, ampliamente utilizadas por consultores y especialistas en hospitales, empresas o escuelas, conllevan un tipo de participación "receptora" (Alastra, Kaneklin \& Scaratti, 2012). El rol de los participantes consiste en adquirir los conocimientos proporcionados, principalmente por medio de la escucha y toma de apuntes, para posteriormente transferir lo aprendido en la práctica. Por su parte, las metodologías de intervención formativa conceptualizan al formador y participantes como co-constructores del conocimiento que conforma la base del desarrollo grupal (Zucchermaglio \& Alby, 2006).

La noción de aprendizaje organizacional asociada a cada modelo, de carácter transferencial para las metodologías lineales y coconstructivo para las metodologías formativas, conlleva implicaciones en lo que atañe al reconocimiento y ejercicio de habilidades duras y blandas involucradas en la realización de las tareas laborales (Heckman, Stixrud \& Urzua, 2006). Las habilidades duras, también llamadas cognitivas, se vinculan a procesos de elaboración de información en el marco de las habilidades del pensamiento (por ejemplo "razonamiento analítico") y del dominio de un campo de conocimiento (por ejemplo "epidemiología"). Las habilidades blandas o no cognitivas aluden a los rasgos de personalidad, de base socio-emocional, que intervienen en la interacción profesional (Rao, 2018), incluyendo aspectos relacionales y comunicativos mediante los que se realiza la actividad colectiva como son la cooperación, la colaboración o la eficacia grupal, entre otros (Cimatti, 2016). En el ámbito de las prácticas laborales del personal sanitario en hospitales, incluyendo entre éstas a la gestión directiva, las habilidades duras han contado con mayor visibilidad al tratarse de un contexto connotado históricamente por el ejercicio de conocimiento altamente especializado, siendo aquellas de tipo blando o relacionales menormente destacadas, particularmente en lo que concierne al ámbito de la formación continua.

Un aspecto crítico ligado a las metodologías lineales consiste en valorar en mayor medida los contenidos a ser transmitidos, difundiendo tácitamente el marco de habilidades duras en detrimento de aquellas de tipo blando. Se discute que estas últimas son lentificadas a partir de una dinámica de intercambio jerárquico y vertical, a la que se sujeta el aprendizaje organizativo desde el enfoque lineal. Una intervención formativa, en contraste, va más allá de los modelos lineales para impulsar un cambio significativo en las modalidades de participación (Engeström, 2011). Para la organización implicada conlleva formular, circular y extender el repertorio de prácticas, promoviendo aprendizaje organizativo a través de la exposición colectiva de 
las competencias y habilidades en uso, y a través del desarrollo, a su vez, de las competencias implicadas en su análisis y discusión. Por medio de estas acciones se activa un proceso reflexivo que requiere del uso de habilidades blandas (como son la resolución de problemas o el trabajo en equipo), reconocidas como componentes clave de procesos de desarrollo y aprendizaje organizacional basado en competencias profesionales (Shandler, 2000).

Las habilidades blandas juegan un rol crucial en la resolución de problemáticas que dependen de la interacción entre profesionales, tarea estrechamente relacionada con la gestión llevada a cabo por directivos en múltiples contextos organizacionales. Se sostiene que la intervención formativa habría de complementar las metodologías lineales, utilizadas predominantemente en la formación continua de directivos y habitualmente circunscritas a núcleos de conocimiento técnico o especializado (Laker \& Powell, 2011), con la finalidad de incluir el reconocimiento de componentes cualitativos de las prácticas de gestión organizacional, entre los que se encuentran las habilidades blandas.

El modelo de Investigación - Intervención Formativa (I-IF de aquí en adelante), adscrito al campo de la psicología cultural de las organizaciones (Engeström, 2015; Vygotsky, 1978; Zucchermaglio, 1996) y entre cuyas metodologías -de carácter neovygotskiano, se encuentran la clínica de la actividad (Clot \& Kostulski, 2011), la psicología performativa aplicada a las organizaciones (Holzman, 2006) o el laboratorio de cambio (LC de aquí en adelante) (Virkkunen \& Newnham, 2013), puede contribuir desde una perspectiva local y situada, al desarrollo de habilidades relacionales y aprendizaje organizacional orientado a la resolución colectiva de problemáticas.
La I-IF cuenta con un doble objetivo; proyectar un proceso formativo para la adquisición y desarrollo de competencias al tiempo en que se sistematizan datos para explorar tanto el funcionamiento y potencial de un grupo laboral en su propio contexto como a la intervención como ámbito de innovación formativa. El proceso de mejora y cambio cualitativo que persigue este modelo es concebido como un incremento progresivo en la construcción de significados y habilidades, cuyo punto de partida y llegada es la experticia profesional.

En México las experiencias posicionadas desde este encuadre, particularmente en relación a organizaciones educativas y del sector salud, se encuentran en fase inicial de desarrollo (Montoro \& Álvarez, 2015; Montoro, 2016; Brito, 2015, 2017a). Existe un reducido número de investigaciones acerca de cómo llevar a cabo prácticas de intervención formativa implementando y evaluando innovaciones metodológicas con el fin de apuntalar habilidades profesionales de distintos tipos.

La I-IF conforma una metodología de soporte o andamiaje, en la que se promueven y negocian innovaciones con la contribución de la comunidad profesional. Su implementación, de acuerdo con Engeström (2011) tiene la viabilidad para, particularmente, renovar procesos de formación y participación practicados en organizaciones y comunidades profesionales del sector salud.

Tal es el caso de las sesiones del Cuerpo de Gobierno (CG de aquí en adelante) de un hospital. En éstas se practican principalmente modelos de gestión, coordinación y cooperación vertical, en correspondencia a la actividad directiva tradicional en hospitales (Engeström, 2018).Tales sesiones conforman un punto de reunión físico y simbólico en el que se entrelazan los resultados de las compe- 
tencias relacionales, como son la integración y la eficacia, y donde los miembros del CG tienen la oportunidad de formarse continuamente a fin de afrontar conjuntamente la gestión del hospital.

No obstante, la estructura y dinámica de las reuniones del $\mathrm{CG}$, caracterizadas por un intercambio jerárquico de información entre director y subalternos, en concordancia con lo anteriormente expuesto, ralentiza la expansión de habilidades y competencias relacionales, haciendo un llamado a métodos participativos encaminados a tal objetivo. La carga de trabajo y capacidad resolutiva del CG interpelan la complejidad y tensión sistémica de su actividad organizativa y son ejemplo de cómo este grupo requiere en modo creciente involucrar lateralmente a sus miembros en acciones formativas de mejora y aprendizaje en el trabajo.

El CG conforma un punto neurálgico en la red de relaciones profesionales del hospital en tanto sistema de actividad mediado por competencias directivas de diversos órdenes. En calidad de equipo conformado por profesionales de diferentes disciplinas (jefes y subjefes de área) cuenta con un nodo de habilidades no siempre visible o conocido por sus propios miembros. En este sentido cabe plantear: ¿Cómo hacer del conocimiento del CG competencias y habilidades existentes? ¿Cómo identificar y estimular áreas de desarrollo y mejora para sus integrantes? ¿Qué técnicas contribuyen al uso y co-construcción de nuevas prácticas formativas orientadas hacia tales objetivos?

En el presente estudio se da cuenta de los resultados parciales de una intervención formativa realizada con directivos del CG de un hospital público mexicano durante mayo y junio de 2017. El carácter general de la intervención consistió en apoyar al CG en el análi- sis de su actividad laboral y funcionamiento como equipo profesional. Los objetivos específicos de la intervención consistieron en incrementar la integración, trabajo colaborativo y mejora de las relaciones inter profesionales como punto de partida para beneficiar el desempeño y eficacia del CG (Montoro \& Brito, 2017; Pereira-Querol et al., 2019). Las actividades realizadas durante esta experiencia consistieron en analizar, discutir y reflexionar acerca de las habilidades profesionales en uso, a fin de avanzar empíricamente en la respuesta a las cuestiones anteriormente señaladas.

Las temáticas abordadas conjuntamente durante las sesiones trataron, en términos generales, acerca del objeto de la actividad del CG (sesión 1), exploración del pasado y presente de la actividad del CG (sesión 2), estado de necesidad para el cambio (sesión 3), problemáticas y alternativas para mejorar la coordinación e integración del CG (sesión 4), monitoreo de cambios en curso y propuesta de realización del shadowing (sesión 5), análisis de resultados del shadowing (sesión $6 \mathrm{y}$ 7).

A partir de la cuarta sesión se exploraron problemáticas cotidianas e insertaron cambios progresivos a fin de favorecer los objetivos de la intervención. Entre tales cambios se incorporó una herramienta orientada a innovar las prácticas formativas del $\mathrm{CG}$; la realización de observaciones tipo shadowing ${ }^{1}$ (Czarniawska, 2007). Esta técnica consiste en el seguimiento que un investigador realiza, como una sombra, para analizar habilidades profesionales de un participante durante una o varias jornadas laborales. Se trata de una técnica observacional adscrita a los métodos de seguimiento, útil para identificar elementos articulatorios de la dinámica organizativa desde un enfoque descriptivo (McDonald, 2005;

\footnotetext{
${ }^{1}$ Se utiliza el anglicismo a lo largo del trabajo, empleado asimismo durante la intervención.
} 
McDonald \& Simpson, 2014).

En el ámbito de los estudios organizacionales cualitativos (Czarniawska, 1992, 2014; Brito, 2017b) tal recurso ha sido provechoso para analizar situacionalmente el liderazgo a través de la identificación de habilidades y estilos de dirección (Noordegraaf, 2014). Su implementación, desde el punto de vista metodológico, no persigue el objetivo de analizar las prácticas laborales desde un posicionamiento interno, como observador interno, sino desarrollar una observación guiada por la búsqueda de exotopia y consecuente configuración de una perspectiva externa, como observador externo (Sclavi, 1989).

Los objetivos del presente estudio consisten en analizar los resultados de esta técnica y llevar a cabo una evaluación metodológica acerca de su funcionamiento y aportación durante la etapa final de la intervención. El shadowing fue considerado por su potencial idoneidad para identificar habilidades relacionales y zonas de desarrollo próximo in situ (ZDP de aquí en adelante). Sin embargo, sus hallazgos no constituyeron un fin en sí mismos, sino un medio para estimular la capacidad de agencia de los participantes, como es requerido en la intervención formativa (Virkkunen \& Ahonen, 2011). La pregunta de investigación que constituye el planteamiento del presente análisis es la siguiente: ¿Ha sido la técnica del shadowing favorable para desarrollar el modelo de intervención formativa -y consecuente fortalecimiento del CG- a través de la identificación de habilidades relacionales y áreas de desarrollo personales? La hipótesis de trabajo, de naturaleza cualitativa de acuerdo con Given (2008), consistió en que el shadowing sería relevante para fortalecer al CG a partir del conocimiento de habilidades existentes e identificación de áreas de desarrollo personales, integrando y desarrollando favorablemente el modelo de intervención formativa.
El trabajo ha constituido una oportunidad para integrar núcleos de conocimiento de primer y segundo orden representados por los datos obtenidos de los seguimientos y aquellos derivados de su discusión durante las sesiones, respectivamente. Para el modelo de intervención formativa, las prácticas discursivas expresadas durante las sesiones escenifican límites, sentidos y significados inherentes al aprendizaje organizativo (Zucchermaglio \& Alby, 2006) . Tales prácticas, interesan por su relación intrínseca con el potencial del CG en tanto grupo laboral, inmerso en un proceso alternativo de formación en la práctica, razón por la cual han constituido el corpus de datos analizado. Al no contar con evidencia empírica respecto a la implementación de la intervención formativa en contextos culturales distintos a las experiencias consolidadas en países europeos o norteamericanos (Sannino, Engeström \& Lahikainen, 2016), el valor de los resultados a exponer consiste en su aportación al debate metodológico en torno al modelo de I-IF a escala internacional. Al parecer, este artículo conforma uno de los primeros estudios (probablemente el primero) en analizar el funcionamiento de la técnica shadowing vinculado al modelo de I-IF en un contexto organizacional latinoamericano (Gonzales-Miranda, Ocampo-Salazar \& Gentilin, 2018).

La contribución del presente trabajo se extiende, por otra parte, al impacto que el propio modelo de I-IF pretende incentivar respecto a la construcción de nuevas modalidades formativas que beneficien las prácticas de los profesionales participantes. En este sentido tanto la intervención como la técnica shadowing contribuyeron al reforzamiento de la comunicación y colaboración intragrupales, apoyando la interconexión de prácticas gestoras del CG. A partir de ello, los directivos del $\mathrm{CG}$ se encontraron en grado de conocer y entrelazar prácticas asociadas con 
su propio desempeño, impactando positivamente en nuevos significados y expansión de su cultura formativa. Los resultados obtenidos ponen en evidencia prácticas discursivas que median el tránsito de un grupo habituado al modelo de intervención lineal hacia uno de carácter formativo, por lo que se aporta una superficie de conocimiento acerca de una innovación metodológica de intervención formativa en la práctica.

\section{Método}

\section{Tipo de investigación}

Se llevó a cabo una I-IF basada en el modelo del LC (Engeström, Virkkunen, Helle, Pihlaja \& Poikela, 1996; Virkkunen \& Newnham, 2013). La I-IF es cualitativa y el tipo de estudio es exploratorio y descriptivo debido al carácter innovador de la metodología formativa empleada. La intervención en su conjunto fue realizada por medio de siete sesiones, videograbadas y transcritas en su totalidad, con una duración promedio de 80 minutos y a razón de una por semana. Las sesiones se integraron a las reuniones semanales del CG.

\section{Participantes y escenario}

Participaron 25 miembros del CG de un Hospital General de Zona con Medicina Familiar en el Estado de Guanajuato, quienes conformaron la muestra del estudio de acuerdo a un criterio de inclusión voluntaria de carácter no probabilístico. Una vez obtenida la autorización para realizar la intervención los miembros del CG acordaron colectivamente su participación. Adicionalmente a las sesiones del LC, se realizó un seguimiento a tres miembros, quienes expresaron voluntariamente su interés en participar; subdirector del hospital, jefa de epidemiología y subdirectora administrativa. La comunidad profesional no contaba con experiencia previa en relación al modelo de la I-IF ni de la técnica observacional utilizada.

\section{Diseño de investigación}

Se llevó a cabo un diseño de investigaciónintervención formativa, de carácter participativo y basado en el modelo del LC en tanto investigación cualitativa aplicada (Sannino, 2011; Laitinen, Sannino \& Engeström, 2016; Virkkunen \& Newnham, 2013).

\section{Instrumentos}

Además de los seguimientos, cuya sistematización se realizó en base a notas de campo de acuerdo con Czarniawska (2007), el corpus de datos se complementó con la transcripción de videograbaciones de la sexta y séptima sesión (análisis de la interacción y habla espontánea) y dos documentos de restitución (Zucchermaglio, Alby, Fatigante \& Saglietti, 2013). Estos fueron elaborados por los participantes individual y anónimamente a fin de evaluar la técnica en función de tres tópicos: 1) contribución al desarrollo profesional personal; b) contribución al desarrollo organizativo del hospital; 3) aspectos positivos y negativos. Por medio de la transcripción de videograbaciones y documentos de restitución se obtuvieron segmentos asociados con la evaluación y percepción del conjunto de participantes en torno a la experiencia.

\section{Procedimiento}

El procedimiento se divide en dos etapas; la primera se relaciona con la realización y análisis de los seguimientos observacionales como parte de la intervención, mientras que la segunda alude al procedimiento de sistematización y análisis de datos para el presente artículo. Se distinguen ambos procedimientos a fin de clarificar dos fases estrechamente relacionadas. 
Primera etapa del procedimiento: Realización y análisis de los seguimientos observacionales.

1. Propuesta, negociación y aceptación voluntaria a participar en los seguimientos (quinta sesión del LC). La duración de cada observación se acordó conjuntamente.

2. Jornadas de seguimiento (efectuadas entre las sesiones cinco y seis del LC). La observación se orientó en base al conjunto de habilidades no cognitivas para el "aprendizaje organizacional centrado en competencias" propuesto por Shandler (2000) (habilidades individuales y de gestión). Cada seguimiento observacional tuvo una duración promedio de ocho horas consecutivas y abarcó los espacios del hospital por donde cada participante realizó sus actividades (tabla 1).

3. Transcripción, sistematización y análisis de las notas de campo del investigador (70 cuartillas) para categorizar habilidades no cognitivas y ZDP (entre las sesiones seis y siete).
4. Presentación de resultados al conjunto de participantes y discusión colectiva (sexta y séptima sesiones).

5. Elaboración de documentos de restitución (séptima sesión).

\section{Segunda etapa del procedimiento: Sistema-} tización y análisis de datos para el presente artículo.

1. Lectura recursiva de las transcripciones (sesiones 6 y 7 de la intervención) y documentos de restitución.

2. Selección de segmentos donde se aludiera claramente a la técnica shadowing y sus resultados.

3. Análisis de los datos por medio de codificación temática, categorización y conteo de frecuencia simple (Richards, 2005), apoyado con el programa MaxQDA. Se incluyeron los resultados obtenidos del shadowing (paso tres de la primera etapa del procedimiento).

4. Definición de dos macro-categorías y correspondientes sub categorías: Macro -categoría 1: Resultados obtenidos de la técnica shadowing; sub categorías: 1.1

Tabla 1

Tiempo de seguimiento observacional y espacio de actividad por participante

\begin{tabular}{lll}
\hline Participante & Horas de seguimiento & Espacios del hospital \\
\hline P1: Subdirector del hospital & 8 horas & Oficina (subdirección) \\
& (turno completo) & Área administrativa \\
& & Urgencias \\
& & Hospitalización (sección de camas) \\
& Salas de espera (hospitalización) \\
& Sección "abastecimiento" \\
P2: Subdirectora & 9 horas & Oficina (jefatura de finanzas y administra- \\
administrativa & (turno completo) & ción) \\
& & Área administrativa \\
P3: Jefa de epidemiologia & 8 horas & Farmacia \\
& (turno completo) & Consultorio de epidemiología \\
& & Salas de espera (especialidades) \\
& & Hospitalización (sección de camas) \\
& & Urgencias \\
\hline
\end{tabular}

Nota: Elaboración propia 
Habilidades no cognitivas en uso (tabla 2); 1.2 ZDP de los participantes (tabla 3). Macro-categoría 2 (tabla 4): Evaluación del shadowing y de la intervención; sub categorías: 2.1 Evaluación de la intervención; 2.2 Evaluación del shadowing; 2.3 Perspectiva de cambio $\mathrm{y}$ mejora.

5. Selección de extractos ejemplares de cada categoría y correspondiente sub categoría. Se seleccionaron fragmentos que contuvieran significados claramente expresados en función del shadowing como técnica y en perspectiva de la intervención como experiencia formativa de orden cualitativo.

\section{Análisis de datos}

Se llevó a cabo un análisis temático, multifocal e interpretativo (Radiker \& Kuckartz, 2020; Flick, 2006) subdividido a razón de las dos fases procedimentales apenas señaladas:

a) Análisis de los datos obtenidos de la observación por medio de la estrategia de codificación de "doble entrada", la cual consideró tanto temáticas pre establecidas como categorías emergentes (Richards, 2005), presentado y discutido durante la intervención (paso tres y cuatro de la primera etapa del procedimiento, respectivamente).

b) Análisis de la evaluación de la técnica observacional por medio de la transcripción de sesiones y documentos de restitución, realizado por medio de la construcción de categorías, codificación y estrategia de selección de extractos propuesta por Emerson, Fretz y Shaw (2011) y Richards (2005) (segunda etapa del procedimiento).

Examinar el funcionamiento del shadowing en vínculo con el modelo de I-IF requirió focalizar la construcción de sentido a partir del punto de vista los participantes en concordancia con la metodología de la investiga- ción situada (Zucchermaglio et al., 2013). La simbología de transcripción es; P1: participante uno, etc.; cursivas: contenido sobresaliente; [...]: omisión de texto; [con texto]: señalamiento del investigador.

\section{Consideraciones éticas}

La intervención, instrumentos y metodología fueron aprobados por el Comité Local de Investigación y Ética del Instituto Mexicano del Seguro Social (IMSS), cuyos particulares se reservan para mantener la confidencialidad y anonimato. Los participantes autorizaron el uso de los datos para fines de investigación por medio de una carta de consentimiento informado mediante la que se dieron a conocer sus derechos como participantes.

Durante cada seguimiento se cumplieron los lineamientos planteados por Johnson (2014) respecto al ejercicio ético y nointrusivo del shadowing en hospitales, respetando la privacidad y protección a pacientes. Ningún dato relacionado con éstos formó parte del desarrollo de la técnica ni de la intervención.

\section{Resultados}

Se sistematizaron dos tipos de resultados por medio de dos macro-categorías. La primera incluye los hallazgos obtenidos de los seguimientos a través de las categorías "habilidades no cognitivas en uso" (tabla 2) y "ZDP de los participantes" (tabla 3). La segunda macro-categoría remite a la evaluación que los participantes manifestaron en relación al shadowing e intervención, incluyendo la perspectiva de cambio y mejora una vez expuestos los resultados iniciales (tabla 4). En todos los casos se señala la frecuencia de segmentos de acuerdo a cada subtema. Las tablas 2 y 3 se presentan asimismo para proporcionar un marco contextual que ejemplifique el núcleo de conocimiento discutido con los participantes durante las sesiones 6 y 7 de la intervención. 
Tabla 2

Resultados del shadowing: habilidades no cognitivas en uso (categoría 1.1)

\begin{tabular}{|c|c|c|c|c|}
\hline Subtemas & $\begin{array}{l}\text { P1. Subdirector } \\
\text { del hospital } \\
(\mathrm{n}=51)\end{array}$ & $\begin{array}{l}\text { P2. Subdirectora ad- } \\
\text { ministrativa } \\
(\mathrm{n}=58)\end{array}$ & $\begin{array}{l}\text { P3. Jefa de epi- } \\
\text { demiologia } \\
(\mathrm{n}=49)\end{array}$ & $\begin{array}{l}\text { Frecuencia } \\
\text { por sub tema } \\
(\mathrm{N}=158)\end{array}$ \\
\hline $\begin{array}{l}\text { 1. Coordinación e inter- } \\
\text { acción con otras áreas }\end{array}$ & 7 & 8 & 7 & 22 \\
\hline 2. Creatividad & 4 & 2 & 0 & 6 \\
\hline 3. Eficiencia & 5 & 6 & 0 & 11 \\
\hline 4. Escucha activa & 2 & 4 & 0 & 6 \\
\hline 5. Gestión de riesgos & 0 & 0 & 12 & 12 \\
\hline $\begin{array}{l}\text { 6. Motivación a los de- } \\
\text { más }\end{array}$ & 6 & 5 & 2 & 13 \\
\hline 7. Motivación personal & 7 & 8 & 2 & 17 \\
\hline 8. Puntualidad & 4 & 5 & 5 & 14 \\
\hline $\begin{array}{l}\text { 9. Resolución de pro- } \\
\text { blemas }\end{array}$ & 9 & 7 & 5 & 21 \\
\hline 10. Responsabilidad & 7 & 8 & 7 & 22 \\
\hline 11. Trabajo en equipo & 0 & 0 & 9 & 9 \\
\hline 12. Tolerancia al estrés & 0 & 5 & 0 & 5 \\
\hline
\end{tabular}

Nota: $\mathrm{N}=$ Total de segmentos analizados, $\mathrm{n}=$ total de segmentos por participante. Frecuencia de segmentos por subtema en cada recuadro, respectivamente. En recuadros sombreados las habilidades con mayor frecuencia en los tres participantes. Fuente: Elaboración propia

En la tabla 2, las frecuencias 22 ron durante la jornada laboral observada. En ("coordinación e interacción con otras áreas" este sentido, por ejemplo, los tres particiy "responsabilidad"), 21 ("resolución de pantes manifestaron la habilidad de coordinar problemas") y 17 ("motivación personal") e interactuar con otras áreas en 7, 8 y 7 representan las habilidades en uso que desta- ocasiones, respectivamente. En contraparte, caron en los tres participantes. Tales ha- se observan asimismo especificidades, donde bilidades corresponden al tipo de actividad de la jefa de epidemiología, por ejemplo, ha sido cada directivo observado (subdirector del la única participante en expresar habilidades hospital, jefa de epidemiología y subdirectora relacionadas con la "gestión de riesgos" duadministrativa) y a las acciones que realiza- rante su actividad laboral. 
Tabla 3

Resultados del shadowing: ZDP de los participantes (categoría 1.2)

\begin{tabular}{lllll}
\hline Subtemas & $\begin{array}{l}\text { P1. Subdirector } \\
\text { del hospital } \\
(\mathrm{n}=38)\end{array}$ & $\begin{array}{l}\text { P2. Subdirectora } \\
\text { administrativa: } \\
(\mathrm{n}=21)\end{array}$ & $\begin{array}{l}\text { P3. Jefa de epi- } \\
\text { demiologia } \\
(\mathrm{n}=34)\end{array}$ & $\begin{array}{l}\text { Frecuencia } \\
\text { tema } \\
(\mathrm{N}=93)\end{array}$ \\
\hline $\begin{array}{l}\text { 1. Habla médica educativa } \\
\text { (incremento en la capacidad } \\
\text { comunicativa con los pacien- } \\
\text { tes). }\end{array}$ & 21 & 0 & 9 & 30 \\
$\begin{array}{l}\text { 2. Gestión de conflictos. } \\
\begin{array}{l}\text { 3. Estilo de liderazgo } \\
\text { coordinación y resolución de } \\
\text { problemas). }\end{array}\end{array}$ & 8 & 19 & 6 & 33 \\
\hline
\end{tabular}

Nota: $\mathrm{N}=$ Total de segmentos analizados, $\mathrm{n}=$ total de segmentos por participante. Frecuencia de segmentos por subtema en cada recuadro, respectivamente. En recuadros sombreados la ZDP propuesta a cada participante.

Fuente: Elaboración propia.

En la tabla 3 se presenta en recuadros sombreados aquellas áreas donde se observaron oportunidades de mejora. En el caso del subdirector del hospital se identificó en 21 ocasiones dificultades en su capacidad comunicativa con los pacientes, mientras que la gestión del conflicto y el estilo de liderazgo conformaron las áreas donde se observó dificultad para la subdirectora administrativa (19 ocasiones) y la jefa de epidemiología (19 ocasiones), respectivamente. Sobresale que cada ZDP corresponde a habilidades no cognitivas que, desde el punto de vista del investigador, podían ser potencialmente reestructuradas desde la lógica de la mejora y desarrollo profesional.

Se llevó a cabo una validación y verificación de autenticidad, coherencia y credibilidad de los resultados (tablas 2 y 3) (Given, 2008) mediante un intercambio realizado entre los participantes del shadowing y el investigador encargado de los seguimientos.

La tabla 4 sintetiza el conjunto de categorías y subtemas identificados en los datos de la transcripción de videograbaciones y documentos de restitución. Como se ha mencio- nado, el presente trabajo integra dos núcleos de conocimiento; el primero se sujeta a los datos obtenidos de los seguimientos (representado en las tablas 2 y 3 ) mientras que el segundo se refiere al resultado de la presentación y discusión de los hallazgos del shadowing durante las sesiones 6 y 7; por esta razón la tabla 4 permite observar el efecto de la circulación de habilidades no cognitivas y ZDP en el discurso de los participantes. Los datos de la tabla 4 fueron validados por medio del criterio de codificación recursiva (Given, 2008) a fin de verificar la consistencia interna, plausibilidad y robustez de los resultados.

Se discute la síntesis de hallazgos de los seguimientos (tablas 2 y 3 ) así como la evaluación de los participantes respecto a la técnica, destacando que la mayor parte de los segmentos analizados aluden a ésta (84 de 106 , tabla 4). Por esta razón se enfocan y discuten extractos relacionados con la percepción de sus "aspectos metodológicos" y respectiva "utilidad" (tablas 5 y 6) por medio de los subtemas más significativos de acuerdo con los objetivos de este artículo. 


\section{Tabla 4}

Evaluación del shadowing y de la intervención

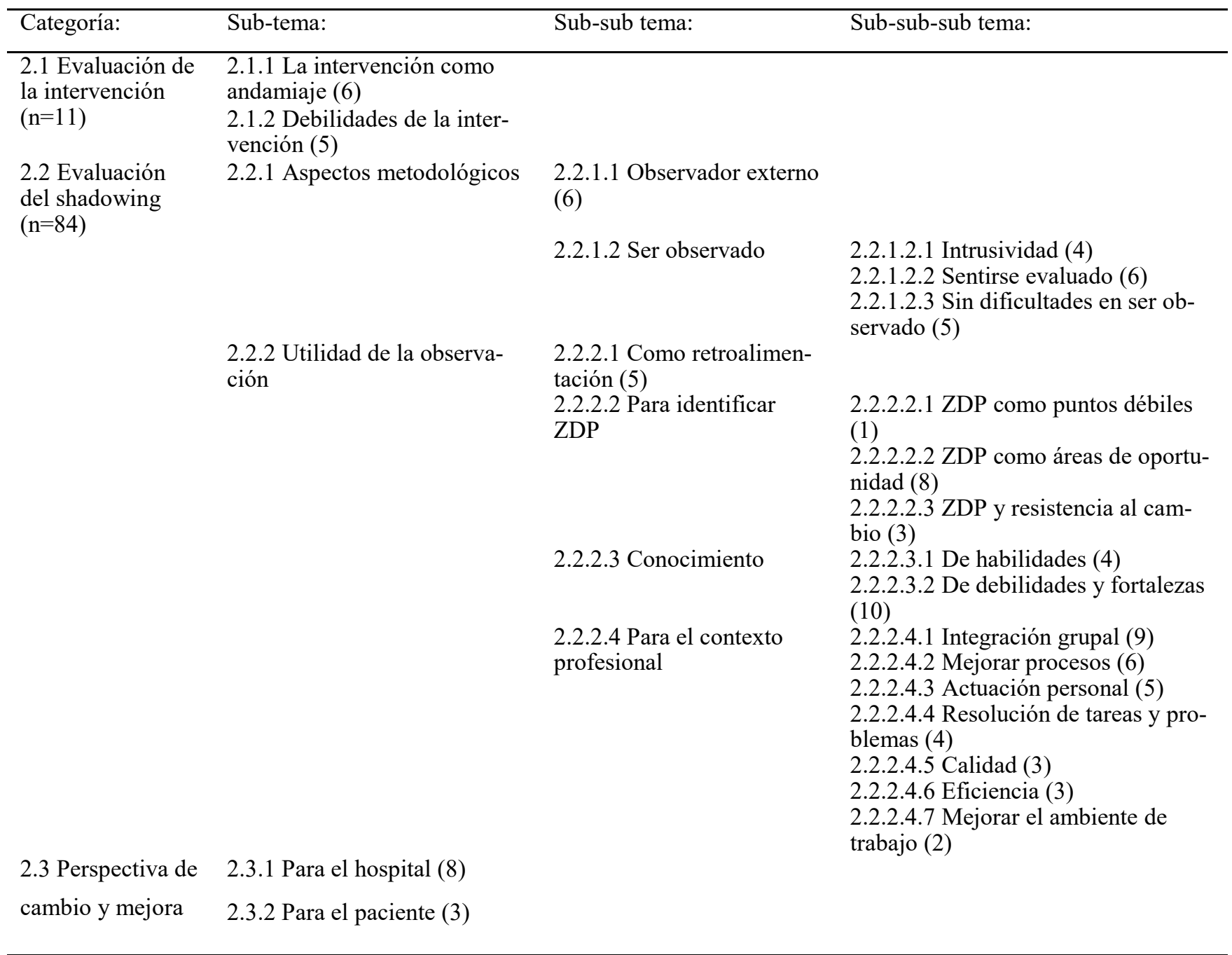

Nota: Total de segmentos analizados $=106 . \mathrm{n}=$ frecuencia de segmentos por categoría. Frecuencia de segmentos por tema entre paréntesis, respectivamente. Fuente: Elaboración propia. 
Tabla 5

Extractos de la categoría 2.2 Evaluación del shadowing y 2.2.1 Aspectos metodológicos

\begin{tabular}{|c|c|c|}
\hline Sub tema & Ext & cto ejemplar \\
\hline \multirow{3}{*}{$\begin{array}{l}2.2 .1 .1 \\
\text { Observador ex- } \\
\text { terno }(6)\end{array}$} & 1 & $\begin{array}{l}\text { P14: Es bueno que alguien externo nos haga observaciones acerca de cómo estamos } \\
\text { realizando nuestro trabajo y dónde podemos mejorar. }\end{array}$ \\
\hline & 2 & $\begin{array}{l}\text { P16: Es un proyecto muy bueno, ya que al ser personas ajenas al hospital, que obser- } \\
\text { van el trabajo diario del personal que labora en el instituto, nos hacen observaciones } \\
\text { que a lo mejor sin darnos cuenta se han dejado de realizar y por muy sencillos o com- } \\
\text { plejos que puedan ser, esto ha afectado en la atención a la salud y en el desempeño } \\
\text { eficaz y eficiente que debe ser nuestro trabajo. }\end{array}$ \\
\hline & 3 & $\begin{array}{l}\text { P9: Siempre es bueno que alguien alejado del problema nos diga cómo estamos, por- } \\
\text { que uno tiene los problemas y cree que lo está haciendo está bien y cree que el otro } \\
\text { está mal, y los demás estamos inmersos en el mismo problema, y no hay quien nos } \\
\text { diga ahí no, o síguele, vas bien. }\end{array}$ \\
\hline \multirow{3}{*}{$\begin{array}{l}\text { 2.2.1.2.1 Intrusi- } \\
\text { vidad (4) }\end{array}$} & 4 & P5: El shadowing intimida. \\
\hline & 5 & P6: Un aspecto negativo; el sentirnos observados, el miedo a cometer errores. \\
\hline & 6 & $\begin{array}{l}\text { P3 [Jefa de Epidemiología]: [...] al principio me sentía nerviosa, me preguntaba ¿qué } \\
\text { va a pasar?, ¿qué voy a hacer con esto?, pero bueno [...] yo creo que máximo a los } \\
\text { veinte minutos me sentí tranquila y cómoda. }\end{array}$ \\
\hline \multirow[t]{3}{*}{$\begin{array}{l}\text { 2.2.1.2.2 } \\
\text { Sentirse evalua- } \\
\text { do (6) }\end{array}$} & 7 & $\begin{array}{l}\text { P9: Contribuye a que la persona quiera mejorar en todos los aspectos de su desarro- } \\
\text { llo profesional, ya que al sentirse observado sabe que se le está realizando una eva- } \\
\text { luación con la finalidad de realizar mejoras a su desempeño. }\end{array}$ \\
\hline & 8 & $\begin{array}{l}\text { P7: Cuando nos sentimos observados es evidente que cambiamos actitudes, pero } \\
\text { está bien que las cambiemos para mejorar, [...] no nada más es una crítica de un com- } \\
\text { pañero a otro o de un superior a otro, pues nos está haciendo un análisis. }\end{array}$ \\
\hline & 9 & $\begin{array}{l}\text { P3 [Jefa de Epidemiología]: [...] cuando alguien se siente observado como que pues a } \\
\text { lo mejor tratamos de mejorar, lo puedo hacer al "ahí se va" pero cuando ahora me } \\
\text { van a supervisar o me va a ver alguien, como que queremos hacerlo mejor. }\end{array}$ \\
\hline \multirow{2}{*}{$\begin{array}{l}\text { 2.2.1.2.3 } \mathrm{Sin} \\
\text { dificultades en } \\
\text { ser observado } \\
\text { (5) }\end{array}$} & 10 & $\begin{array}{l}\text { P9: No veo aspectos negativos sólo que la persona por su criterio no quiera que le } \\
\text { realicen o no lo acepte. }\end{array}$ \\
\hline & 11 & $\begin{array}{l}\text { P10: No veo ningún aspecto negativo, ya que es un proyecto que hace reflexión y } \\
\text { mejoras de acuerdo a capacidades. }\end{array}$ \\
\hline
\end{tabular}

Nota: Frecuencia de segmentos por tema entre paréntesis, respectivamente. Extractos numerados consecutivamente. Fuente: Elaboración propia.

En resumen, los hallazgos más relevantes respecto al shadowing atañen a la "coordinación e interacción con otras áreas" como habilidad no cognitiva persistente en los tres participantes y ZDP identificadas para cada uno ("habla médica educativa", "gestión de conflictos" y "estilo de liderazgo"). Respecto a la discusión e interpretación derivada de la participación conjunta en las sesiones destaca la subcategoría "2.2 Evaluación del shadowing" $(\mathrm{n}=84)(\mathrm{y}$ respectivos extractos) como mapa emergente de significados colectivos. 
Tabla 6

Extractos de la categoría 2.2 Evaluación del shadowing y 2.2.2 Utilidad de la observación

\begin{tabular}{|c|c|c|}
\hline Sub tema & \multicolumn{2}{|c|}{ Extracto ejemplar } \\
\hline $\begin{array}{l}\text { 2.2.2.2.2 ZDP } \\
\text { como áreas de }\end{array}$ & 1 & $\begin{array}{l}\text { P1 [Subdirector del hospital]: Visualiza de manera directa y descubre áreas de oportunidad } \\
\text { personales. }\end{array}$ \\
\hline oportunidad (8) & 2 & P15: Nos enseña áreas de oportunidad que no se tenían visualizadas. \\
\hline \multirow[t]{2}{*}{$\begin{array}{l}\text { 2.2.2.3.1 Conoci- } \\
\text { miento "de habi- } \\
\text { lidades" (4) }\end{array}$} & 3 & $\begin{array}{l}\text { P19: A través de esta técnica me permitió identificar las competencias como directivos, por } \\
\text { ejemplo, mencionamos aquí que hace falta trabajo en equipo, que son las competencias } \\
\text { que un directivo podría tener para poder realizar su trabajo más eficientemente [...] la ges- } \\
\text { tión del conflicto es otro tipo de competencia que identifiqué [...] a través de esta técnica } \\
\text { se pasa a lo real y podemos confirmar que esas competencias tenemos que tenerlas, desa- } \\
\text { rrollarlas para poder trabajar para que se incrementen, para eso me sirvió, también, identi- } \\
\text { ficar esas competencias directivas. }\end{array}$ \\
\hline & 4 & $\begin{array}{l}\text { P13: Contribuye al conocimiento de las habilidades de cada miembro del cuerpo de go- } \\
\text { bierno. }\end{array}$ \\
\hline $\begin{array}{l}\text { 2.2.2.3.2 Conoci- } \\
\text { miento "de debi- } \\
\text { lidades y fortale- }\end{array}$ & 5 & $\begin{array}{l}\text { P16: Nos ayuda para ver las cosas que se realizan de manera adecuada y eficiente para } \\
\text { poder seguirlas poniendo en práctica, de igual manera mejorarlas y las cosas o hábitos que } \\
\text { no son adecuados para la realización del trabajo, dejarlas de hacer. }\end{array}$ \\
\hline zas" (10) & 6 & P20: Nos señala nuestros errores, actitudes positivas y actitudes negativas. \\
\hline \multirow[t]{2}{*}{$\begin{array}{l}2.2 .2 .4 .1 \text { Integra- } \\
\text { ción grupal (9) }\end{array}$} & 7 & $\begin{array}{l}\text { P15: [...] nos ha permitido analizar nuestro funcionamiento como cuerpo de gobierno, cómo } \\
\text { estamos integrados, analizar nuestros defectos y virtudes que nos permitan un mejor fun- } \\
\text { cionamiento laboral. }\end{array}$ \\
\hline & 8 & $\begin{array}{l}\text { P5: Fue de gran ayuda, nos servirá para integrar un buen equipo de trabajo, todo esto para } \\
\text { mejores resultados en calidad de atención al derechohabiente y en los procesos del hospi- } \\
\text { tal. }\end{array}$ \\
\hline \multirow[t]{2}{*}{$\begin{array}{l}\text { 2.2.2.4.3 Actua- } \\
\text { ción personal (5) }\end{array}$} & 9 & $\begin{array}{l}\text { P5: Recibes un análisis no crítico y el análisis te ayuda a mejorar como persona y tu calidad } \\
\text { en el servicio y la atención. }\end{array}$ \\
\hline & 10 & $\begin{array}{l}\text { P1 [Subdirectora administrativa]: Si el individuo sujeto de shadowing aplica los conceptos o } \\
\text { recomendaciones para mejorar, su contribución a la mejora del grupo será inminente ya } \\
\text { que forma parte del hospital. }\end{array}$ \\
\hline $\begin{array}{l}\text { 2.2.2.4.4 Resolu- } \\
\text { ción de tareas y }\end{array}$ & 11 & $\begin{array}{l}\text { P13: Nos permite crecer y fortalecer de manera colectiva la resolución de tareas, activida- } \\
\text { des y problemática existente. }\end{array}$ \\
\hline problemas (4) & 12 & $\begin{array}{l}\text { P4: Favorece el desarrollo profesional colectivo, si nosotros vamos mejorando en la resolu- } \\
\text { ción de problemas eso se refleja en el hospital y en la mejora de éste. }\end{array}$ \\
\hline $\begin{array}{l}\text { 2.2.2.4.6 Eficien- } \\
\text { cia (3) }\end{array}$ & 13 & $\begin{array}{l}\text { P8: Permite desarrollar habilidades para realizar las actividades de manera eficiente, permi- } \\
\text { te identificar las competencias directivas que se tienen que fortalecer para eficientar las } \\
\text { actividades como jefes de área. }\end{array}$ \\
\hline
\end{tabular}

Nota: Frecuencia de segmentos por tema entre paréntesis, respectivamente. Extractos numerados consecutivamente. Fuente: Elaboración propia. 


\section{Discusión}

Las habilidades identificadas por medio de los seguimientos (tabla 2), de acuerdo con Shandler (2000), remiten al ámbito individual $\mathrm{y}$ de gestión de procesos. Respecto a la dimensión individual destaca la comunicación, gestión del conflicto y creatividad, lo cual conforma un núcleo consistente de habilidades no cognitivas. En cuanto a la gestión de procesos, de menor densidad, sobresale la coordinación entre áreas y la gestión de riesgos. En concordancia con los estudios observacionales sobre liderazgo y prácticas directivas en contexto (Czarniawska, 2007), tal superficie de datos interpela competencias en uso, confrontando los nodos de habilidades prescriptivas característicos de buena parte de la literatura de gestión organizacional, comparable con lo expuesto por Garrat (1987) y Luthans (1992). Más que presentar un marco de habilidades que los directivos del CG deberían poseer, los resultados indican atributos del repertorio existente de habilidades y prácticas de dirección, mismos que derivan de la experticia directiva.

La densidad de hallazgos da cuenta asimismo de habilidades propias al liderazgo situacional de acuerdo con Northouse (2013), mismas que permiten a los participantes observados, en calidad de directivos del CG, llevar a término tareas desde las que se articula la actividad colectiva del hospital. Si bien dichas habilidades representan atributos individuales, su principal función es la de ser relacionales, es decir, mediante éstas los participantes establecen vínculos operativos, de base socioemocional, con otros profesionales (Bruni \& Gherardi, 2007).

Northouse (2013) señala que el liderazgo situacional es aquel que responde a la demanda y capacidad de adaptación en función de tareas específicas, lo cual es extrapolable al ejercicio directivo observado. Las ZDP identificadas se vinculan en este sentido con las tareas efectivamente realizadas y se sujetan al rol directivo en cuestión (tabla 3). Desde este ámbito la técnica shadowing contribuyó a la delimitación de áreas de mejora desde una perspectiva situacional, señalando aspectos de las tareas cotidianas de acuerdo al rol ejercido (en tanto jefes y subjefes de área) y habilidades propias a este sector laboral (Ellström \& Ellström, 2018). Al hacerlo, la ZDP propuesta a cada participante (habla médica educativa, gestión de conflictos y estilo de liderazgo) se supeditó a la dinámica, espacios, rol y actividades expresadas durante la jornada de observación.

Puesto que la intervención, desarrollo de seguimientos y posterior exposición de resultados constituyeron una innovación y actividad formativa no dominante para los miembros del CG, la exteriorización de puntos de vista relacionados con su ejercicio conformó un aspecto crucial. De acuerdo con Sannino (2008b, p. 332) las innovaciones en una intervención formativa son demandantes para los participantes puesto que estos deben construir continuidad entre lo existente y lo nuevo. Por esta razón los extractos de las tablas 5 y 6, asociados con los resultados anteriores, significan posicionamientos epistémicos ubicados entre límites de las actividades formativas dominantes y no dominantes para el CG.

En tanto núcleo de un resultado de segundo orden, propio al ámbito de la investigación aplicada, tales sub temas conforman un punto referencial en la emergencia de significados, particularmente en lo relacionado con el desplazamiento de la cultura formativa en este hospital.

La exposición y discusión de datos obtenidos del shadowing fue más allá de su carácter extractivo, conformándose como un estímulo para la intervención y desarrollo del 
$\mathrm{CG}$, como es requerido en el LC (Virkkunen \& Newnham, 2013). Al proveer conocimiento acerca de las propias habilidades y perspectivas de mejora, los participantes contaron con un "espejo" desde el cual visualizar al CG como grupo profesional (ídem). Ello contribuyó a superar los límites de los contenidos "descontextualizados" de los modelos de intervención lineal mayormente practicados en hospitales (Engeström, 2018).

Al exteriorizar una valoración positiva en torno al shadowing como técnica, los participantes refrendaron componentes teóricometodológicos señalados por Sclavi (1989) y Czarniawska (2007); 1) pertinencia de un observador externo; 2) lejanía del observador; 3 ) no adscripción del mismo a la institución participante (extractos 1, 2 y 3, tabla 5). Su realización respondió adecuadamente al problema del encapsulamiento observacional intra grupal, donde la inclusión de un punto de análisis externo por parte del investigador, como $o b$ servador externo y no como observador interno, fue percibido positivamente. Tal resultado respalda la discusión propuesta por Bruskin (2019) de acuerdo a la cual el rol del investigador es efectivamente asignado por la organización durante el estudio, en este caso alineado a los objetivos de la técnica shadowing. Tales datos coinciden con los hallazgos identificados por Erlingsdottir, Ersson, Borell y Rydenfält (2018), de acuerdo a los cuales un proceso de innovación formativa con directivos requiere que el investigador desarrolle efectivamente un rol de soporte externo para promover la iniciativa de cambio a partir del reconocimiento de problemáticas, visible en los extractos de la tabla 5 (sub tema 2.2.1.1) y 6 .

Por otra parte, considerando el corpus de segmentos que refieren a la observación (84 de 106, tabla 4), destaca la baja frecuencia de valoraciones asociadas con su intrusivi- dad (cuatro) (subtema 2.2.1.2.1), contrastado por cinco extractos en los que se manifestó no percibir alguna dificultad para llevar a cabo los seguimientos (subtema 2.2.1.2.3). El shadowing no fue valorado preponderantemente, en este sentido, como un ejercicio observacional intrusivo, aun teniendo lugar en una institución cuyas prácticas profesionales no son comúnmente accesibles a proyectos de investigación de carácter etnográfico, observacional o cualitativo, como sostiene Johnson (2014). Tal valencia conforma un respaldo empírico a favor del empleo de una técnica de observación no canónica en contextos hospitalarios.

En contraparte, el mayor grado de criticidad se aprecia en la ponderación de la técnica en términos de "sentirse evaluado", "ser analizado" o "supervisado" mientras se es observado (extractos 7, 8 y 9, tabla 5). El hecho de que ésta fuera interpretada como una experiencia mediante la que se mejoran las propias tareas al ser observado, refleja parte de la cultura institucionalizada de supervisión y señalamiento de fallos (práctica dominante). Tal aspecto resulta problemático al confrontarse con la naturaleza de la actividad observacional propuesta, pues ésta fue planteada como un recurso para identificar habilidades no cognitivas y ZDP (práctica no dominante).

Aunque ello indica un efecto no deseado (elocuente del discutido efecto Hawthorne o modificación de la conducta a consecuencia de saberse observado), señala que la inserción de tal metodología, de carácter reflexivo y descriptivo, estimuló parcialmente el desmantelamiento de atributos contrapuestos a su planteo original. Por esta razón, en tal conjunto de extractos se manifiesta un habla y discurso transicional cotejable con lo discutido por Sannino (2008b), derivado del ejercicio observacional como innovación metodológica y participativa. 
El conjunto de extractos de la categoría "evaluación del shadowing" representa por lo tanto un resultado intermedio, como indican Zucchermaglio y Alby (2006), puesto que los segmentos incluyen tanto aspectos de la epistemología sedimentada del CG (representados por la "intrusividad" de la técnica y "sentirse evaluado") como apertura a una técnica no practicada hasta entonces (manifestada en la valoración positiva del "observador externo" y la baja densidad de "dificultades" en ser observado).

Finalmente, se identifica apertura del horizonte de observación profesional (Zucchermaglio, 2013) e indicios de posicionamientos agentivos (Virkkunen, 2006; Sannino, 2008a) como ejes articulatorios de la utilidad de la técnica shadowing (tabla 6).

El horizonte de observación apela al margen de conocimiento con que cuentan los actores en una organización para acceder a las habilidades y competencias de otros miembros. Tal horizonte atañe a la visualización de "ZDP como áreas de oportunidad" (extractos 1 y 2, tabla 5), "conocimiento de habilidades" (extractos 3 y 4, tabla 5) y "conocimiento de debilidades y fortalezas" (extractos 5 y 6, tabla 5). Tales segmentos confirman la función articulatoria del shadowing como técnica de "observación en movimiento" (Czarniawska, 2007), cuyos hallazgos activaron una plataforma de conocimiento que permitió visibilizar y transparentar habilidades y ZDP distribuidas en el sistema de actividad laboral (Zucchermaglio, 2013).

El acceso a tal conocimiento medió el cumplimiento de uno de los principales objetivos del modelo de I-IF: estimular el desarrollo de la capacidad de agencia del grupo participante (Virkkunen, 2006). Puesto que la agencia consiste, siguiendo a Virkkunen, en la expansión del horizonte de observación e iniciativa para transformar la actividad laboral
(2006, p. 49), los extractos 7 al 13 (tabla 6) exteriorizan la capacidad para llevar a cabo transformaciones en el propio funcionamiento y continuar desarrollándose a consecuencia del ejercicio observacional. Tales posicionamientos se desdoblan, desde una perspectiva individual y colectiva, en ámbito de la "integración grupal” (extractos 7 y 8 ), "actuación personal" (extractos 9 y 10) e incremento en la "resolución de tareas" y "eficiencia" (extractos 11, 12 y 13). Un aspecto común entre tales segmentos consiste en vincular los resultados de la observación con la mejora, crecimiento, eficiencia y calidad de las prácticas del CG. Tales segmentos dan cuenta de la disposición transformacional compartida, la cual, de acuerdo con McSherry y Pearce (2016), conforma la base para la mejora de la calidad de los servicios en materia de atención a la salud a partir del desarrollo de competencias de liderazgo y fortalecimiento de relaciones inter grupales de un equipo profesional.

Se observan, asimismo, indicios de disposición al cambio y reconocimiento de la propia capacidad de agencia -objetivo del modelo del LC como metodología de I-IF-, desarrollo de las prácticas de gestión y coordinación del $\mathrm{CG}$, particularmente visible en las voces de P3 ("nos ha permitido analizar nuestro funcionamiento como cuerpo de gobierno, cómo estamos integrados, analizar nuestros defectos y virtudes que nos permitan un mejor funcionamiento laboral", extracto 7) y P8 ("permite desarrollar habilidades para realizar las actividades de manera eficiente [...] para eficientar las actividades como jefes de área", extracto 13, tabla 5).

En suma, tres indicadores de carácter cualitativo y no paramétrico, permiten responder afirmativamente al planteamiento de investigación e hipótesis de trabajo del presente estudio. En primera instancia, se com- 
prueba la pertinencia del shadowing para sistematizar conocimiento acerca de ZDP y habilidades relacionales existentes; la superficie de datos construida por medio de los seguimientos da cuenta de 158 segmentos asociados con habilidades blandas presentes en la cotidianeidad laboral y sujetas a las tareas de cada participante observado (tabla 2). Respecto a la ZDP, el shadowing fue útil para producir 93 segmentos por medio de los que se identificó el área de oportunidad para la mejora personal en base a conocimiento situado (30 segmentos en promedio por participante, tabla 3).

En segunda instancia, la alta incidencia de segmentos asociados con la técnica shadowing como parte de la intervención corrobora su impacto en términos de recursividad discursiva sobresaliente (95 segmentos de 106, categorías 2.2 y 2.3, tabla 4). En último lugar se observa manifestación transversal de valencia positiva respecto al shadowing en los extractos incluidos en la categoría 2.2 "Evaluación del shadowing" (tabla 4); en ésta 60 de 84 segmentos expresan un sentido favorable al uso de la técnica observacional en cuestión.

El total de segmentos (tablas 2-4), ponderados desde una óptica vinculante, representan evidencia consistente para afirmar que el shadowing fue favorable a la implementación del modelo de intervención formativa a través de la identificación de habilidades relacionales y áreas de desarrollo personales, posibilitando su discusión colectiva. Tal discusión incidió, a su vez, en la integración del equipo profesional participante; al tiempo en que el CG analizó los hallazgos de los seguimientos puso en práctica, a su vez, habilidades blandas que interpelan al aprendizaje organizacional cualitativo, como son la colaboración y reflexión conjunta (aspectos transversales de la sub categoría 2.2.2.4, utilidad "para el contexto profesional", tabla 4).

\section{Conclusiones y limitaciones}

Se concluye que la técnica shadowing y respectiva discusión de hallazgos favoreció la co -construcción de una práctica coherente con la intervención formativa, contribuyendo positivamente a la articulación del modelo. Los resultados indican prácticas discursivas que median el proceso mediante el que un grupo profesional sin experiencia al respecto movilizó concepciones tradicionales y produjo significados transicionales a partir de una actividad formativa no dominante. La contribución del artículo, en este sentido, consiste en presentar datos que dan cuenta del desenvolvimiento inicial de la I-IF en un grupo novicio. Sin embargo, se detecta que ello es insuficiente para el conocimiento de ulteriores etapas vinculadas con el desarrollo y consolidación del modelo de I-IF. Así, una problemática emergente concierne a las tensiones que el discurso de los participantes podría incluir en otras fases, como es el caso de las resistencias o dilemas producidos al realizar transformaciones de mayor calado en el sistema de actividad (Brito, 2017a). El presente estudio conforma un paso necesario hacia tal dirección y aporta elementos que respaldan la coconstrucción de una práctica de formación innovadora.

Al ser este uno de los primeros estudios en evaluar el vínculo entre shadowing e I-IF a escala internacional, una de las principales limitaciones recae en la confirmación de resultados de investigaciones que hubieran sido realizadas en condiciones similares. Desde esta óptica, como señalan Sannino, Engeström y Lahikainen (2016), los datos discutidos representan un punto de partida para avanzar en tal dirección, representando un recurso para investigadores interesados en implementar el modelo de intervención formativa y avanzar en la obtención de nuevo conocimiento al respecto. 
Como se ha señalado, operativizar y explorar analíticamente el funcionamiento de cambios e innovaciones metodológicas formativas conforma un campo que aún no se encuentra plenamente desarrollado. A tal efecto, otra limitación del estudio consiste en no enfocar dificultades técnicas y metodológicas tanto de los seguimientos como del modelo de intervención formativa. Tales dificultades incluyen la capacitación del investigadorinterventor, el proceso de negociación para la autorización del proyecto, la construcción de la relación entre investigador y participantes, la dificultad de categorizar los datos de la observación y diseñar las sesiones en que serán discutidos, así como los desafíos para el cumplimiento de directrices éticas al llevar a cabo los seguimientos en los distintos espacios del hospital.

A fin de consolidar resultados se detecta la necesidad de obtener datos respecto a las habilidades blandas de un mayor número de participantes, y su respectiva discusión, a fin de caracterizar en profundidad el patrimonio de recursos profesionales asociados a la gestión organizativa. Ello ayudará a superar el nivel exploratorio que ha definido al presente estudio. Otra limitación que debe señalarse atañe al desconocimiento del impacto de la intervención respecto a la mejora de la actividad colectiva del hospital por lo que se enfatiza que el presente estudio interpela únicamente al ámbito de las prácticas del CG como grupo participante.

Finalmente, debido al estado inicial de los estudios de I-IF en México y Latinoamérica (Pereira-Querol et al., 2019), es necesario implementar el modelo de I-IF y la técnica shadowing en otros hospitales y contextos laborales, con el objetivo de obtener un mayor número de observaciones y experiencias, en perspectiva longitudinal y a mayor escala. La consolidación de una nueva generación de estudios beneficiará la socialización del modelo y su inserción, así como la recolección de evidencias empíricas para conocer a fondo su utilidad y dificultades.

Es importante y necesario que organizaciones del sector salud, y de otros tipos, incluyan modelos de intervención formativa para apoyar a los profesionales en el fortalecimiento de sus habilidades y competencias, equilibrando las experiencias concentradas en el modelo de intervención lineal. En este sentido se señala que los esfuerzos en la realización de este tipo de investigación han de continuar, preservando como principal orientación que los resultados apoyen la resolución conjunta de problemáticas, logro colectivo de metas y mejora de la calidad del servicio.

\section{Agradecimientos}

El autor agradece a C. R. Montoro Sanjosé (Universidad de Guanajuato) por la coordinación de la investigación (PI) y a Y. Engeström (University of Helsinki) y A. Sannino (University of Tampere) por su colaboración durante la misma. Se agradece al equipo de asistentes conformado por A. T. Álvarez Bravo, J. G. Mendoza Guerrero y A. V. Rojas Medina (Universidad de Guanajuato). Agradecimiento especial al Cuerpo de Gobierno del hospital y al Instituto Mexicano del Seguro Social.

\section{Financiamiento}

La investigación contó con financiamiento de la Universidad de Guanajuato por medio del proyecto P0084.601.017 de la Convocatoria Institucional UG 2016-2017.

\section{Referencias}

Alastra, V., Kaneklin, C., \& Scaratti, G. (2012). La formazione situata. Milán: AIF - Franco Angeli.

Brito, H. (2015). La teoría de la actividad como recurso para la intervención y gestión del cambio organizativo: el caso del contexto escolar. En Ramírez, G. et al., (Eds.). El análisis organiza- 
cional en México y América Latina. Retos y perspectivas a 20 años de Estudios (455-494). México: REMINEO.

Brito, H. (2017a). Habla resistiva e intervención formativa en una organización escolar. Polis, 13 (2), 73-105.

Brito, H. (2017b). Implicaciones metodológicas del shadowing en la investigación organizacional. En De la Rosa, A., Rosas, J., Núñez, C. \& Espinosa, E. (Coords.). Estudios organizacionales y administración: Perspectivas de estudio (271-310). México: REMINEO.

Bruni, A., \& Gherardi, S. (2007). Studiare le pratiche lavorative. Bolonia: Il Mulino.

Bruskin, S. (2019). Insider or outsider? Exploring the fluidity of the roles through social identity theory. Journal of Organizational Ethnography, 8(2), 159-170. doi:10.1108/JOE-09-2017 $-0039$

Cimatti, B. (2016). Definition, development, assessment of soft skills and their role for the quality of organizations and enterprises. International Journal for Quality Research. 10(1), 97 -130. doi:10.18421/IJQR10.01-05

Clot, Y., \& Kostulski, K. (2011). Intervening for transforming: The horizon of action in the Clinic of Activity. Theory \& Psychology, 21 (5), 681-696. doi:10.1177/0959354311419253

Czarniawska, B. (1992). Exploring complex organizations. A Cultural Perspective. Newbury ParkLondon - New Delhi: SAGE.

Czarniawska, B. (2007). Shadowing and other techniques for doing fieldwork in modern societies. Mälmo: Liber - Copenhagen Business School Press.

Czarniawska, B. (2014). Why I think shadowing is the best field technique in management and organization studies. Qualitative Research in Organizations and Management: An International Journal, 9(1), 90 - 93. doi:10.1108/ QROM-02-2014-1198

Ellström, E., \& Ellström, P. (2018). Two modes of learning-oriented leadership: a study of firstline managers. Journal of Workplace Learning. 30(7), 545-561. doi:10.1108/JWL-032018-0056

Emerson, R.M., Fretz, R.I., \& Shaw, L. (2011). Writing ethnographic field notes. Chicago: University of Chicago Press.

Engeström, Y. (2011). From design experiments to formative interventions. Theory \& Psychology, 21(5), 598-628. doi:10.1177/0959354311419252
Engeström, Y. (2015). Learning by expanding: An activity-theoretical approach to developmental research. Cambridge: Cambridge University Press.

Engeström, Y. (2018). Expertise in transition. Expansive learning in medical work. Cambridge: Cambridge University Press. doi:10.1080/07294360.2018.1441809

Engeström, Y., Virkkunen, J., Helle, M., Pihlaja, J., \& Poikela, R. (1996). Change laboratory as a tool for transforming work. Lifelong Learning in Europe, 1(2), 10-17.

Erlingsdottir, G., Ersson, A., Borell, J., \& Rydenfält, C. (2018). Driving for successful change processes in healthcare by putting staff at the wheel. Journal of Health Organization and Management, 32(1), 69 - 84. doi:10.1108/ JHOM-02-2017-0027

Flick, U. (2006). An introduction to qualitative research. Londres: SAGE.

Garrat, B. (1987). The learning organization and the need for directors who think. Aldershot: Gower.

Given, L. (2008). The SAGE encyclopedia of qualitative research methods. Londres: SAGE.

Gonzales-Miranda, D. R., Ocampo-Salazar, C. A., \& Gentilin, M., (2018). Organizational Studies in Latin America. A Literature Review (20002014). Innovar, 28(67), 89-109. doi:10.15446/ innovar.v28n67.68615

Heckman, J., Stixrud, J., \& Urzua S. (2006). The effects of cognitive and noncognitive abilities on labor market outcomes and social behavior. Journal of Labor Economics, 24(3), 411482. doi:10.1086/504455

Holzman, L. (2006). Lev Vygotsky and the new performative psychology: some implications for business and organizations. En Hosking, D. M. \& McNamee, S. (Ed.). The social construction of organization (254-268). Ljubljana: Liber - Copenhagen Business School Press.

Johnson, B. (2014). Ethical issues in shadowing research. Qualitative Research in Organizations and Management: An International Journal, 9 (1), 21 - 40. doi:10.1108/QROM-09-20121099

Laitinen, A., Sannino, A., \& Engeström, Y. (2016). From controlled experiments to formative interventions in studies of agency: methodological considerations. Educação, 39(núm. especial), 14-23. doi:10.15448/19812582.2016.s. 24321 
Laker, D., \& Powell, J. (2011). The differences between hard and soft skills and their relative impact on training transfer. Human Resource Development Quarterly, (22), 111-122. doi:10.1002/hrdq.20063

Luthans, F. (1992). Organizational behaviour. New York: McGraw-Hill.

McDonald, S. (2005). Studying actions in context: a qualitative shadowing method for organizational research. Qualitative Research, 5(4), 455 - 473. doi:10.1177/1468794105056923

McDonald, S., \& Simpson, B. (2014). Shadowing research in organizations: the methodological debates. Qualitative Research in Organizations and Management: An International Journal, 9(1), 3 - 20. doi:10.1108/QROM-022014-1204

McSherry R., \& Pearce P. (2016). What are the effective ways to translate clinical leadership into heath care quality improvement? Journal of Healthcare Leadership. 8, 1-7. doi: 10.2147/ JHL.S46170

Montoro, C. (2016). Learn or earn? Making sense of language teaching and learning at a Mexican university through a Change Laboratory intervention. Learning, Culture and Social Interaction, (11), 48-57. doi: 10.1016/ j.lcsi.2016.05.001

Montoro, C., \& Álvarez, A. (2015). Activitytheoretical analysis and interpretation of Change Laboratory data. En Lengeling, M. \& Mora, P. (Eds.). Perspectivas sobre la investigación cualitativa (535-547). Guanajuato: Universidad de Guanajuato.

Montoro, C., \& Brito, H. (2017). Crossing cultures: A healthcare Change Laboratory intervention in Mexico [Full paper]. Trabajo presentado en el 33rd EGOS Colloquium, Copenhagen, Dinamarca.

Noordegraaf, M. (2014). Shadowing managerial action instead of recording managerial text. Qualitative Research in Organizations and Management: An International Journal, 9(1), 41 - 46. doi:10.1108/QROM-02-2014-1197

Northouse, P. (2013). Leadership: theory and practice. Thousand Oaks: SAGE.

Pereira-Querol, M. A., Beltran, S. L., Montoro, C., Valenzuela, I., Castro, W., Tresserras, E., \& Esteve, O. (2019). Intervenciones formativas en educación y aprendizaje en el trabajo: aplicaciones del Laboratorio de Cambio en Iberoamérica. Revista Internacional de Educación y Aprendizaje, 7(2), 83-96. doi:10.37467/ gka-revedu.v7.1992
Radiker, S., \& Kuckartz, U. (2020). Análisis de datos cualitativos con MAXQDA: Texto, audio, video. Berlin: MAXQDA Press.

Rao, M. (2018). Soft skills: toward a sanctimonious discipline. On the Horizon, 26(3), 215224. doi:10.1108/OTH-06-2017-0034

Richards, L. (2005). Handling Qualitative Data. A Practical Guide. Londres: SAGE Publications.

Sannino, A. (2008a). From talk to action: experiencing interlocution in developmental interventions. Mind, Culture, and Activity, 15(3), pp. 234257. doi:10.1080/10749030802186769

Sannino, A. (2008b). Sustaining a non-dominant activity in school: Only an utopia? Journal of educational change, (9), 329-338. doi:10.1007/ s10833-008-9080-z

Sannino, A. (2011). Activity theory as an activist and interventionist theory. Theory \& Psychology, 21(5), 571-597. doi:10.1177/0959354311417485

Sannino, A., Engeström, Y., \& Lahikainen, J. (2016). The dialectics of authoring expansive learning: tracing the long tail of a Change Laboratory. Journal of Workplace Learning, 28(4), 245-262. doi:10.1108/JWL-01-2016-0003

Sclavi, M. (1989). A una spanna da terra. Milán: Feltrinelli.

Shandler, D. (2000). Competency and the learning organization. Menlo Park: CRISP.

Virkkunen, J. (2006). Dilemmas in building shared transformative agency. Activités, 3(1), 43-66. doi:10.4000/activites. 1850

Virkkunen, J., \& Ahonen, H. (2011). Supporting expansive learning through theoretical-genetic reflection in the Change Laboratory. Journal of organizational change management, 24(2), 229-243. doi:10.1108/09534811111119780

Virkkunen, J., \& Newnham, D. (2013). The Change Laboratory. A tool for collaborative development of work activities. Rotterdam: Sense publishers.

Vygotsky L. (1978). Mind in society. The development of higher psychological processes. Londres: Harvard University Press.

Zucchermaglio, C. (1996). Vygostkij in azienda. Apprendimento e comunicazione nei contesti di lavoro. Roma: Carocci.

Zucchermaglio, C. (2013). Cognizione al lavoro. Milán: EULED.

Zucchermaglio, C., \& Alby, F. (2006). Psicologia culturale delle organizzazioni. Roma: Carocci.

Zucchermaglio, C., Alby, F., Fatigante, M. \& Saglietti, M. (2013). Fare ricerca situata in psicología sociale. Bolonia: Il Mulino. 